\title{
Erratum
}

\section{Erratum to "Some Fixed Point Theorems of Integral Type Contraction in Cone Metric Space"}

\author{
Farshid Khojasteh, ${ }^{1}$ Zahra Goodarzi, ${ }^{2}$ \\ and Abdolrahman Razani ${ }^{2,3}$ \\ ${ }^{1}$ Department of Mathematics, Science and Research Branch, Islamic Azad University, Tehran 14778, Iran \\ ${ }^{2}$ Department of Mathematics, Faculty of Science, Imam Khomeini International University, \\ Qazvin, 34149-16818, Iran \\ ${ }^{3}$ School of Mathematics, Institute for Research in Fundamental Sciences, \\ P.O. Box 19395-5746, Tehran, Iran
}

Correspondence should be addressed to Farshid Khojasteh,fr_khojasteh@yahoo.com

Received 20 December 2010; Accepted 5 January 2011

Copyright (C) 2011 Farshid Khojasteh et al. This is an open access article distributed under the Creative Commons Attribution License, which permits unrestricted use, distribution, and reproduction in any medium, provided the original work is properly cited.

We regret making following mistake in the above-mentioned paper [1]. We would like to correct it and explain some notations.

(1) In [1] we introduced a new concept of integral type contraction in cone metric spaces and generalized Brancieri and Meir-Keeler theorems in such spaces. [1, Theorem 2.9] is an extension of Brancieri's theorem, and [1, Theorem 3.2] is an extension of Brancieri and Meir-Keeler's results. We asserted the following in [1, Theorem 2.9].

(i) "Let $(X, d)$ be a complete cone metric space and $P$ be a normal cone. Suppose $\phi$ : $P \rightarrow P$ is a non-vanishing map and a sub-additive cone integrable on each $[a, b] \subset$ $P$ such that for each $\epsilon \gg 0, \int_{0}^{e} \phi d_{p} \gg 0$. If $f: X \rightarrow X$ is a map such that for all $x, y \in X$

$$
\int_{0}^{d(f(x), f(y))} \phi d_{p} \leq \alpha \int_{0}^{d(x, y)} \phi d_{p}
$$

for some $\alpha \in(0,1)$, then $f$ has a unique fixed point in $X . "$

Also, we asserted in [1, Theorem 3.2] the following.

(ii) "Let $(X, d)$ be a complete regular cone metric space and $f$ be a mapping on $X$. Assume that there exists a function $\theta$ from $P$ into itself satisfying the following:

(B1) $\theta(0)=0$ and $\theta(t) \gg 0$ for all $t \gg 0$. 
(B2) $\theta$ is nondecreasing and continuous function. Moreover, its inverse is continuous.

(B3) For all $0 \neq \epsilon \in P$, there exists $\delta \gg 0$ such that for all $x, y \in X$

$$
\theta(d(x, y))<\epsilon+\delta \text { implies } \theta(d(f x, f y))<\epsilon .
$$

(B4) For all $x, y \in X$

$$
\theta(x+y) \leq \theta(x)+\theta(y)
$$

Then $f$ has a unique fixed point."

After this theorem, we asserted the following in [1, Remark 3.3] that:

(iii) "If $\phi: P \rightarrow P$ is a non-vanishing map and a sub-additive cone integrable on each $[a, b] \subset P$ such that for each $\epsilon \gg 0, \int_{0}^{\epsilon} \phi d_{p} \gg 0$ and $\theta(x)=\int_{0}^{x} \phi d_{P}$, then $\theta$ is satisfies in all conditions of [1, Theorem 3.2]. Equivalently [1, Theorem 2.9] is concluded from [1, Theorem 3.2]."

Note that, in (B2) of [1, Theorem 3.2] and [1, Remark 3.3], we have emphasized that the map $\theta(x)=\int_{0}^{x} \phi d_{P}$ must have the continuous inverse, but unfortunately this assumption has been forgotten mistakenly in [1, Theorem 2.9]. Note that this assumption is a necessary condition to prove [1, Theorem 2.9].

(2) To prove [1, Theorem 3.2] and [1, Theorem 2.9], it is sufficient that $\theta(x)=\int_{0}^{x} \phi d_{P}$ satisfy the following: for each sequence $\left\{x_{n}\right\} \subset P$

$$
\theta\left(x_{n}\right) \longrightarrow 0 \text { implies } x_{n} \longrightarrow 0 \text {. }
$$

On the other hand, (4) is equivalent to continuity of $\theta^{-1}$ at zero.

(3) In [2] the authors gave a counterexample on [1, Theorem 2.9] only for our misprint that we have asserted it in the above as you have seen. They also gave a comment for us at the end of their paper to correct such misprint and emphasized that $\theta$ must have the continuous inverse. As you have seen, we have asserted and emphasized such note in (B2) of [1, Theorem 3.2] and [1, Remark 3.3] before the authors in [2] mentioned it.

Nevertheless, we do apologize to the readers for this mistake.

\section{Acknowledgment}

The third author would like to thank the School of Mathematics of the Institute for Research in Fundamental Sciences, Teheran, Iran, for supporting this research (Grant no. 89470126).

\section{References}

[1] F. Khojasteh, Z. Goodarzi, and A. Razani, "Some fixed point theorems of integral type contraction in cone metric spaces," Fixed Point Theory and Applications, vol. 2010, Article ID 189684, 13 pages, 2010.

[2] I. D. Arandelović and D. J. Kečkić, "A counterexample on a theorem by Khojasteh, Goodarzi, and Razani," Fixed Point Theory and Applications, vol. 2010, Article ID 470141, 6 pages, 2010. 